\title{
On the possibility of applying severe plastic deformation by high pressure torsion for the manufacture of Al-Nb metal matrix composites
}

\author{
G. R. Khalikova ${ }^{\dagger, 1,2}$, G. F. Korznikova ${ }^{1}$, K. S. Nazarov ${ }^{1}$, R. Kh. Khisamov ${ }^{1}$, S. N. Sergeev ${ }^{1}$, \\ R. U. Shayakhmetov ${ }^{1}$, R. R. Mulyukov ${ }^{1,2}$ \\ ${ }^{\dagger}$ gulnara.r.khalikova@gmail.com \\ ${ }^{1}$ Institute for Metals Superplasticity Problems RAS, 39 S. Khalturin St., Ufa, 450001, Russia \\ ${ }^{2}$ Ufa State Petroleum Technological University, 1 Kosmonavtov St., Ufa, 450062, Russia
}

\begin{abstract}
The article discusses the possibility of fabrication an aluminium based matrix composite of the Al-Nb hybrid system using severe plastic deformation by high pressure torsion (HPT). Disks of aluminum (with diameters from 6 to $12 \mathrm{~mm}$ ) and niobium (12 $\mathrm{mm}$ in diameter), were stacked in the form of a three-layer Al-Nb-Al package, and subjected to deformation. HPT was carried out at room temperature on Bridgman anvils under a pressure of $5 \mathrm{GPa}$ at $N=10,25$ and 30 revolutions, at a strain rate of $\omega=1$ and $2 \mathrm{rpm}$. Regardless of the choice of the aluminum disk diameter and the deformation modes, monolithic and defect-free samples were fabricated. However, the most effective fragmentation and distribution of niobium in the aluminum matrix was observed when a diameter of aluminum discs was $10 \mathrm{~mm}$ and deformation modes $N=25$ and 30 revolutions and $\omega=2 \mathrm{rpm}$ were applied. Three structural areas were found in the processed samples: the central one with wide curved layers of niobium in aluminum, a finely dispersed lamellar structure in the mid radius area, and a uniform distribution of niobium particles in aluminum matrix at the periphery. The observed heterogeneity of the structure in the samples correlated well with changes in microhardness, the values of which varied nonmonotonically: the minimum level (about $100 \mathrm{HV}$ ) was observed in the center, the maximum values (about 280 and $300 \mathrm{HV}$ ) in the middle of the radius and about $130 \mathrm{HV}$ at the periphery of the sample, for $N=25$ and 30 revolutions, respectively. In addition, it was shown by X-ray diffraction methods that at a higher strain rate and $N=25$ and 30, strain-induced aging occurred in the composite material with the synthesis of the hardening intermetallic phase $\mathrm{Al}_{3} \mathrm{Nb}$, the volume fraction of which increased from 2.8 to $3.1 \%$ with increasing number of revolutions.
\end{abstract}

Keywords: metal matrix composite, aluminum, niobium, high pressure torsion, nanostructure.

УДК: 669, 54-162.2, 54-19

\section{О возможности применения интенсивной пластической деформации кручением под высоким давлением для изготовления Al-Nb металломатричных композиционных материалов}

\author{
Халикова Г. Р. ${ }^{\dagger, 1}$, , Корзникова Г.Ф. ${ }^{1}$, Назаров К.С. ${ }^{1}$, Хисамов Р. Х. ${ }^{1}$, Сергеев С. Н. ${ }^{1}$, \\ Шаяхметов Р. У. ${ }^{1}$, Мулюков Р. Р. ${ }^{1,2}$ \\ ${ }^{1}$ Институт проблем сверхпластичности металлов РАН, ул. С. Халтурина, 39, Уфа, 450001, Россия \\ ${ }^{2}$ Уфимский государственный нефтяной технический университет, ул. Космонавтов, 1, Уфа, 450062, Россия
}

В статье рассматривается возможность формирования алюмоматричного композиционного материала гибридной системы Al-Nb путем интенсивной пластической деформацией кручением (ИПДК) под высоким давлением. Деформированию подвергали дисковые заготовки алюминия (диаметром от 6 до 12 мм) и ниобия (диаметром 12 мм), 
уложенные в виде трехслойного пакета Al-Nb-Al. ИПДК осуществляли при комнатной температуре на наковальнях Бриджмена под давлением 5 ГПа на $N=10,25$ и 30 оборотов, при скорости деформации $\omega=1$ и 2 об/мин. Исследования показали, что независимо от выбора диаметра алюминиевого диска и режима деформации формируются монолитные и бездефектные образцы. Однако наиболее эффективное дробление и распределение ниобия в матрице алюминия наблюдаются при диаметре алюминиевых заготовок 10 мм и режимах деформации $N=25$ и 30 оборотов и $\omega=2$ об/мин. При этом в образцах имеют место три структурные области: центральная с широкими изогнутыми слоями ниобия в алюминии, на середине радиуса - тонкодисперсная пластинчатая структура и на периферии однородное распределение частиц ниобия в алюминиевой матрице. Наблюдаемая гетерогенность структуры в образцах хорошо коррелирует с изменениями микротвердости, значение которой варьируются немонотонно: в центре наблюдается минимальный уровень (около $100 \mathrm{HV}$ ), на середине радиуса - максимальные значения (около 280 и $300 \mathrm{HV}$ ) и на периферии образца около $130 \mathrm{HV}$, соответственно для $N=25$ и 30 оборотов. Кроме того, методами рентгеноструктурного анализа показано, что при большей скорости деформации и $N=25$ и 30 в композиционном материале протекает деформационно-индуцированное старение с синтезом упрочняющей интерметаллидной фазы $\mathrm{Al}_{3} \mathrm{Nb}$, объемная доля которой возрастала с 2.8 до $3.1 \%$ с ростом числа оборотов.

Ключевые слова: металломатричный композит, алюминий, ниобий, кручение под давлением, наноструктура.

\section{1. Введение}

Для создания алюмоматричных композиционных материалов, с точки зрения приближения системы взаимодействия армирующей фазы и Al матрицы к термодинамически стабильной, активно применяются различные методы на основе принципа in situ. При этом в $\mathrm{Al}$ матрице синтезируются упрочняющие интерметаллидные (армирующие) фазы за счет их зарождения и роста, которые имеют меньшую деградацию при повышенных температурах [1]. Возможности изготовления алюмоматричных композитов по принципу in situ опробовано такими методами как, например, механическое легирование [2], разновидности быстрой кристаллизации [3], сварка трением с перемешиванием [4], интенсивная пластическая деформация (ИПД) [5] и др. Из перечисленных методов интенсивная пластическая деформация наиболее активно применяется для изготовления объемных наноструктурных металломатричных композитов [6].

В последнее время ведутся исследования по формированию слоистых металломатричных композитов из отдельных дисков двух разнородных металлов через ИПД, реализованное кручением под высоким давлением при комнатной температуре, например, $\mathrm{Al}-\mathrm{Mg}$ [7], $\mathrm{Al}-\mathrm{Cu}$ [8], $\mathrm{Ag}-\mathrm{Cu}$ [9], $\mathrm{Zn}-\mathrm{Mg}$ [10]. В этом случае образуются гибридные системы, в которых, после значительных изменений в микроструктуре за счет протекания диффузионных процессов, формируются уникальные многоуровневые структуры.

Алюмоматричный композит на основе гибридной системы Al-Nb является перспективным для исследований, т.к. температура плавления ниобия в 2.5 раза выше, чем у чистого $\mathrm{Al}$, и растворимость $\mathrm{Nb}$ в $\mathrm{Al}$ в твердом состоянии составляет около 0.05 вес.\%, что приводит к наибольшему вкладу в уровень механических свойств от синтезируемых упрочняющих интерметаллидных фаз. Возможности изготовления композитов на основе бинарной системы Al-Nb с дисперсными упрочняющими частицами $\mathrm{Al}_{3} \mathrm{Nb}$ успешно реализованы, например, быстрой кристаллизацией [11] и сваркой трением с перемешиванием [12].
В связи с вышеизложенным, целью настоящей работы является оценка возможности применения интенсивной пластической деформации кручением под высоким давлением, как метода in situ изготовления алюмоматричного композита гибридной системы Al-Nb.

\section{2. Материал и методики исследований}

В качестве исходных материалов служат прутки из чистого $\mathrm{Al}(99.5$ вес.\%) и $\mathrm{Nb}$ чистотой 99 вес.\%. Исходные дисковые образцы вырезаются из прутков электроискровым методом и механически утоняются до толщины 0.5 мм. При этом диаметр ( $)$ дисковых образов из $\mathrm{Al}$ варьируется от 6 до 12 мм, диаметр дисков из $\mathrm{Nb}$ составляет 12 мм. Для изготовления образцов металломатричного композита, заготовки $\mathrm{Al}$ и $\mathrm{Nb}$ укладываются в виде трехслойного пакета Al-Nb-Al.

Интенсивная пластическая деформация кручением (ИПДК) под высоким давлением осуществляется при комнатной температуре (RT) на наковальнях Бриджмена под давлением 5 ГПа на $N=10,25$ и 30 оборотов, при скорости деформации $\omega=1$ и 2 об/мин.

Исследования структуры проводятся на поперечном диаметральном срезе образцов с помощью сканирующего электронного микроскопа (CЭM) Tescan Mira 3LMH используя детектор обратно-рассеянных электронов. Рентгеноструктурный анализ проводится методом Ритвелда на $\mathrm{Cu}_{\mathrm{Ka}}$-излучении с узким параллельным рентгеновским пучком повышенной интенсивности, которое позволяет определять долю фаз с точностью не менее $0.1 \%$. Аттестация микротвердости алюмоматричного композита осуществляется с использованием твердомера Affri DM8A при нагрузке 10 г. Измерения микротвердости проводятся на поперечном сечении вдоль диаметра диска. Статистическая обработка результатов выполняется с доверительной вероятностью 95\%.

\section{3. Результаты и их обсуждение}

Согласно серии ранних работ $[8,13]$, формирование монолитных металломатричных композитов при укладке слоев чистого $\mathrm{Al}$ и $\mathrm{Cu}$ в виде трехслойных паке- 
тов $\mathrm{Al}-\mathrm{Cu}-\mathrm{Al}$ и $\mathrm{Cu}-\mathrm{Al}-\mathrm{Cu}$ путем ИПДК имеет место при оптимальном давлении около 5 ГПа, $\omega=1$ об/мин и $N=10$. При этом диаметр заготовок $\mathrm{Al}$ и $\mathrm{Cu}$ составляет 12 мм, толщина около 0.5 мм. Аналогичные режимы ИПДК и геометрия исходных заготовок из чистых $\mathrm{Al}$ и $\mathrm{Nb}$ применяются к деформации пакета $\mathrm{Al}-\mathrm{Nb}-\mathrm{Al}$. При этом формируются монолитные и бездефектные образцы. Однако интенсивного перемешивания слоев не наблюдается, и по образцу сохраняется слоистость с некоторым их дроблением на периферии образца (Рис. 1). Для более значительного дробления и перемешивания исходных слоев $\mathrm{Al}$ и $\mathrm{Nb}$ в процессе ИПДК рассматриваются варианты деформации пакета $\mathrm{Al}-\mathrm{Nb}-\mathrm{Al}$ из исходных заготовок $\mathrm{Al}$ различного диаметра $(6,7$, 8 и 10 мм) и толщины при условии сохранения объема материала (толщина заготовок увеличивается при уменьшении их диаметра). При этом геометрия заготовок из $\mathrm{Nb}$ остается неизменной. Вариации диаметров $\mathrm{Al}$ диска, который заведомо меньше, чем исходный диск из $\mathrm{Nb}$, обусловлены повышением технологической пластичности в процессе деформации осадкой пакета Al-Nb-Al.

На Рис. 1 приведены структуры полированных торцевых поверхностей деформированных образов Al-Nb-Al с различным исходным диаметром $\mathrm{Al}$ диска при идентичных режимах ИПДК и укладки слоев. Видно, что удовлетворительного перемешивания слоев $\mathrm{Al}$ и $\mathrm{Nb}$ также не происходит, хотя отличия в распределении исходных компонентов все же наблюдаются. Так, с увеличением диаметра Al диска дробление усиливается, a распределение $\mathrm{Al}$ и $\mathrm{Nb}$ в образце становится более однородным.

Согласно [14], повышение скорости деформации при ИПДК приводит к росту доли вовлеченных в деформацию систем скольжения в материале. Это способствует, например, формированию равноосных нанокристаллических зерен $\gamma$-фазы в аустенитной нержавеющей стали, в результате протекания деформационно-индуцированного как прямого $\left(\gamma \rightarrow \alpha^{\prime}\right)$, так и обратного $\left(\alpha^{\prime} \rightarrow \gamma\right)$ превращений, в то время как при малых скоростях деформации имеет место формирование удлиненных субзерен $а$-фазы только за счет действия прямого деформационно-индуцированного превращения [15]. В нашем случае, повышение скорости деформации при ИПДК приводит к более значительному дроблению и лучшему распределению $\mathrm{Nb}$ в образце (Рис. 1).

Для дальнейшего увеличения степени измельчения и перемешивания слоев $\mathrm{Al}$ и $\mathrm{Nb}$ осуществлена деформация образцов Al-Nb-Al до 25 и 30 оборотов. При этом получены монолитные деформированные образцы без видимых трещин и сколов. В этом случае имеет место интенсивное дробление и перемешивание слоев $\mathrm{Al}$ и $\mathrm{Nb}$ практически во всем объеме (Рис. 1), что согласуется c [10]. Однако, несмотря на увеличение количества оборотов, в центре обоих образцов степень перемешивания не столь значительна, как на середине радиуса и периферии, и по-прежнему наблюдаются отдельные (дисперсные) четко выраженные слои и включения $\mathrm{Nb}$ в $\mathrm{Al}$ (Рис. 1). С повышением количества оборотов при деформации с 25 до 30 центральная область минимально проработанной структуры сужается. $\varnothing \mathrm{Al}=12 \mathrm{~mm}, N=10, \omega=1 \mathrm{rpm}$

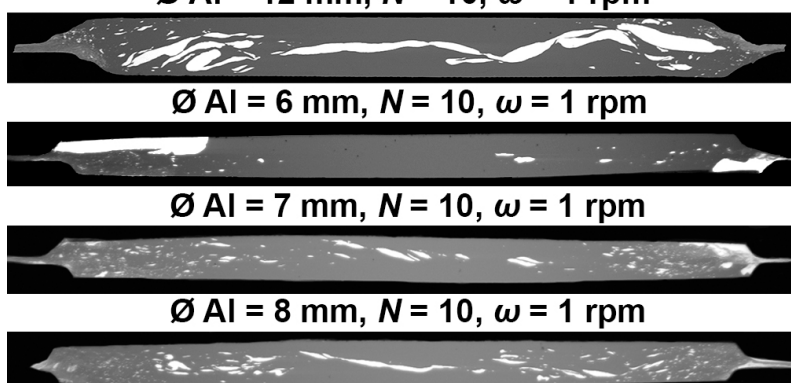

$\varnothing \mathrm{Al}=10 \mathrm{~mm}, N=10, \omega=1 \mathrm{rpm}$

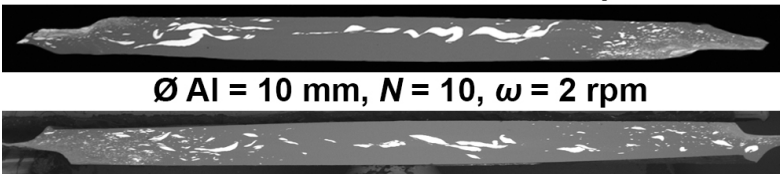

$\varnothing \mathrm{Al}=10 \mathrm{~mm}, N=25, \omega=2 \mathrm{rpm}$

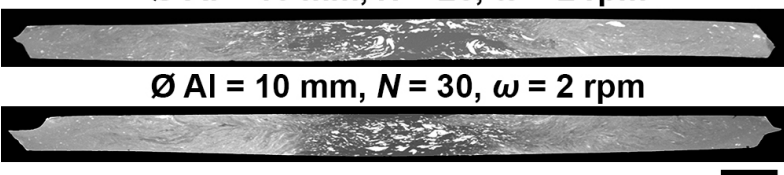

$1 \overline{\mathrm{mm}}$

Рис. 1. СЭМ изображения полированных поперечных сечений образцов $\mathrm{Al}-\mathrm{Nb}-\mathrm{Al}$ с различными исходными диаметрами (Ø) Al диска и режимами ИПДК (количество оборотов $N$ и скорость деформации $\omega$ ). Темный контраст соответствует чистому алюминию, светлый контраст - ниобию.

Fig. 1. SEM images of polished cross sections of Al-Nb-Al samples with different initial diameters $(\varnothing)$ of an Al disk and HPT conditions (revolutions $N$ and rotation speed $\omega$ ). Dark contrast is pure aluminum, bright contrast is niobium.

Следует отметить, что в процессе деформации в результате ламинарного течения композиционного материала в пакете $\mathrm{Al}-\mathrm{Nb}-\mathrm{Al}$ обоих образцов формируются вихревые структуры. В центре образцов имеют место широкие изогнутые слои исходного материала (Рис. $2 \mathrm{a}, \mathrm{d}$ ). На середине радиуса исходный материал трансформируется в тонкодисперсную полосчатую структуру толщиной наблюдаемых слоев не более 100 нм (Рис. 2 b, е). На периферии образцов формируется смешанная структура, состоящая из отдельных областей тонкодисперсных прослоек и однородной составляющей (Рис. $2 \mathrm{c}, \mathrm{f}$ ). При этом увеличение количества оборотов при деформации с 25 до 30 существенно расширяет области однородной структуры при сохранении единичных зон с полосчатой составляющей.

Описанные выше структурные изменения обусловлены неоднородностью деформации по сечению образца при ИПДК трехслойного пакета Al-Nb-Al, которая зависит от расстояния: чем дальше от центра образца к его периферии, тем величина деформации больше [14]. Также, очевидно, что с увеличением числа оборов $N$ возрастает величина деформации, которая приводит к более интенсивному дроблению и перемешиванию исходного слоя ниобия в алюминии.

Образование вихревых структур во время ИПДК наблюдается и анализируется в различных материалах, включая дуплексную нержавеющую сталь, эвтектоидный сплав Zn-22 вес.\% Al, алюминий высокой чистоты 


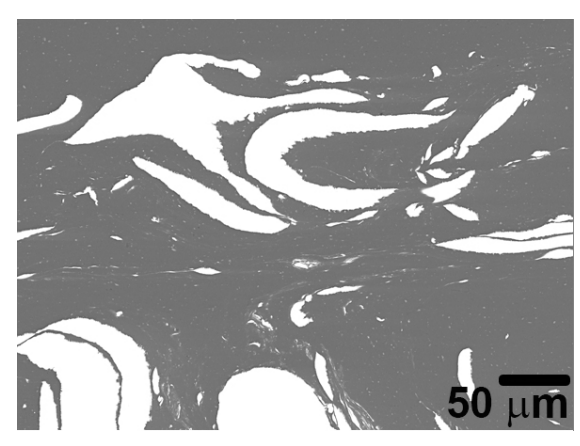

a

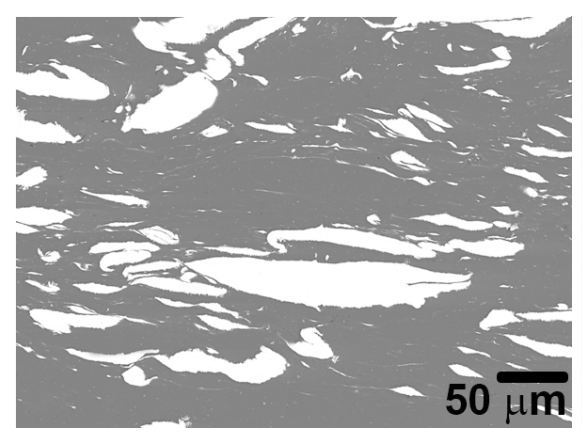

d

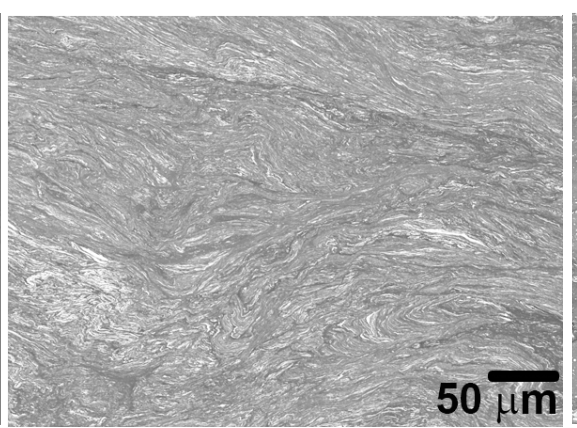

$\mathrm{b}$

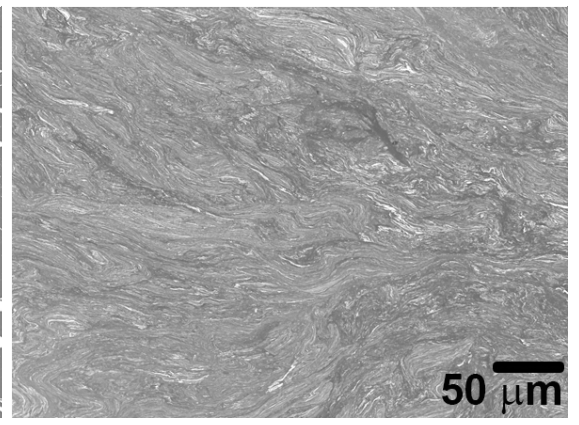

e

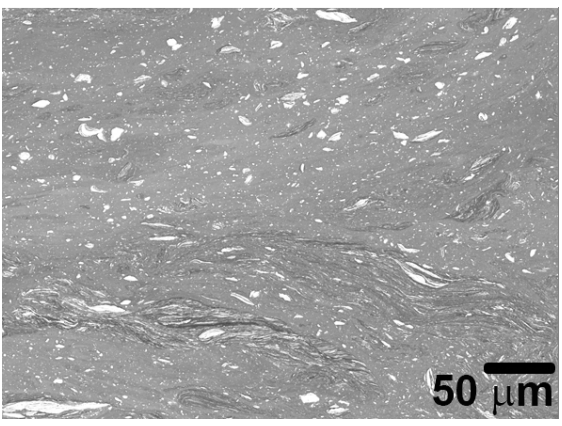

C

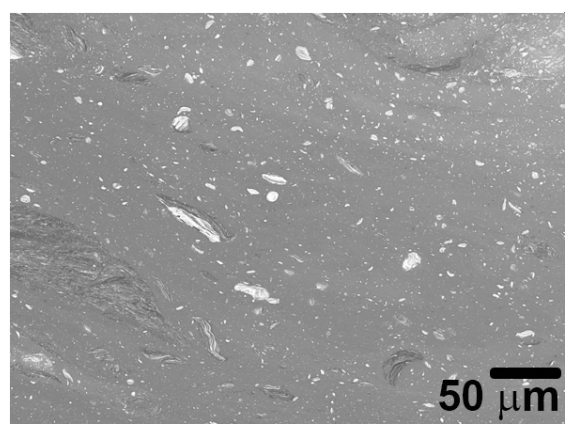

$\mathrm{f}$

Pис. 2. Типичные микроструктуры композита $\mathrm{Al}-\mathrm{Nb}-\mathrm{Al}$, изготовленного ИПДК при $N=25$ и $\omega=2$ об/мин (а-с), $N=30$ и $\omega=2$ об/мин (d-f): в центре $(\mathrm{a}, \mathrm{d})$; на середине радиуса $(\mathrm{b}, \mathrm{e})$; на периферии $(\mathrm{c}, \mathrm{f})$ образцов (темный контраст соответствует чистому алюминию, светлый контраст - ниобию).

Fig. 2. Typical microstructures of Al-Nb-Al composite, processed by HPT at $N=25$ and $\omega=2 \mathrm{rpm}(\mathrm{a}-\mathrm{c}), N=30$ and $\omega=2 \mathrm{rpm}$ (d-f): in the center $(a, d)$; in the middle of the radius (b, e); at the edge (c, f) of the samples (dark contrast is pure aluminum, bright contrast is niobium).

(99.99\%) и в объемных металлических стеклах [16-18]. Подобные неустойчивости к изгибу или складыванию имеют место и в других процессах пластического сдвига твердых тел, таких как трение и сдвиговая деформация изогнутых тел, и могут быть объяснены образованием вихревоподобных складчатых неустойчивостей, которые могут появляться на границе раздела между двумя разнородными жидкостями, движущимися с разной скоростью $[18,19]$.

Согласно [20], при нормальных условиях в бинарной системе Al-Nb в твердом состоянии возможно формирование $\mathrm{AlNb}_{3}, \mathrm{AlNb}_{2}$ и $\mathrm{Al}_{3} \mathrm{Nb}$ фаз. В работе [21] приведены расчетные показатели энтальпии, из которых следует, что наиболее отрицательным значением обладает фаза $\mathrm{Al}_{3} \mathrm{Nb}$, которая является наиболее устойчивым соединением и образуется в первую очередь. В нашем случае, из результатов проведенного рентгеноструктурного анализа следует, что деформация образцов Al-Nb-Al при $N=10$ и $\omega=2$ об/мин способствует формированию гибридной системы без упрочняющих фаз (Рис. 3 a). В то время как, при ИПДК пакетов $\mathrm{Al}-\mathrm{Nb}-\mathrm{Al}$, прокрученных при $N=25$ и $N=30$ и $\omega=2$ об/мин на рентгенограммах появляются пики от $\mathrm{Al}_{3} \mathrm{Nb}$ фазы (Рис. 3 b, с). При этом увеличение N c 25 до 30 (при прочих равных условиях) приводит к росту объемной доли $\mathrm{Al}_{3} \mathrm{Nb}$ фазы с 2.8 до 3.1\%. Т.е. в процессе ИПДК при комнатной температуре и $N \geq 25$ имеет место образование пересыщенного ниобием алюминиевого твердого раствора с последующим протеканием деформационно- индуцированного старения и синтезом частиц упрочняющей фазы $\mathrm{Al}_{3} \mathrm{Nb}$.

Структурные изменения в алюмоматричном композите $\mathrm{Al}-\mathrm{Nb}-\mathrm{Al}$, которые имеют место при ИПДК с $\omega=2$ об/мин, хорошо согласуются с результатами измерений микротвердости (Рис. 4). Так, при $N=10$ в центре и на середине радиуса образца, где неоднородно располагаются крупные включения и слои $\mathrm{Nb}$ в $\mathrm{Al}$, значения микротвредости соизмеримы с показателями в деформированном состоянии $\mathrm{Al}$ образца и составляют около 75 HV. На периферии образца, где располагаются дисперсные включения $\mathrm{Nb}$ в $\mathrm{Al}$, микротвердость достигает значений около $110 \mathrm{HV}$. Увеличение количества оборотов, в целом, приводит к росту микротвердости. При этом ход кривых изменения микротвердости по диаметру образца для $N=25$ и $N=30$ идентичен. В центре образца, где структура прорабатывается в наименьшей степени и сохраняются отдельные четко выраженные дисперсные слои и включения $\mathrm{Nb}$ в $\mathrm{Al}$, микротвердость минимальна и составляет около $100 \mathrm{HV}$ для обоих состояний. С ростом степени деформации, т.е. по мере удаления от центра образца к его периферии, значения микротвердости сначала возрастают, достигая максимума около 280 и $300 \mathrm{HV}$ (соответственно для $N=25$ и $N=30$ ) на середине радиуса, где имеет место тонкодисперсная пластинчатая микроструктура, а затем уменьшаются до значений около $130 \mathrm{HV}$ (для $N=25$ и $N=30)$ на периферии образца, где преобладает наиболее однородное распределение $\mathrm{Nb}$ в $\mathrm{Al}$ матрице. 


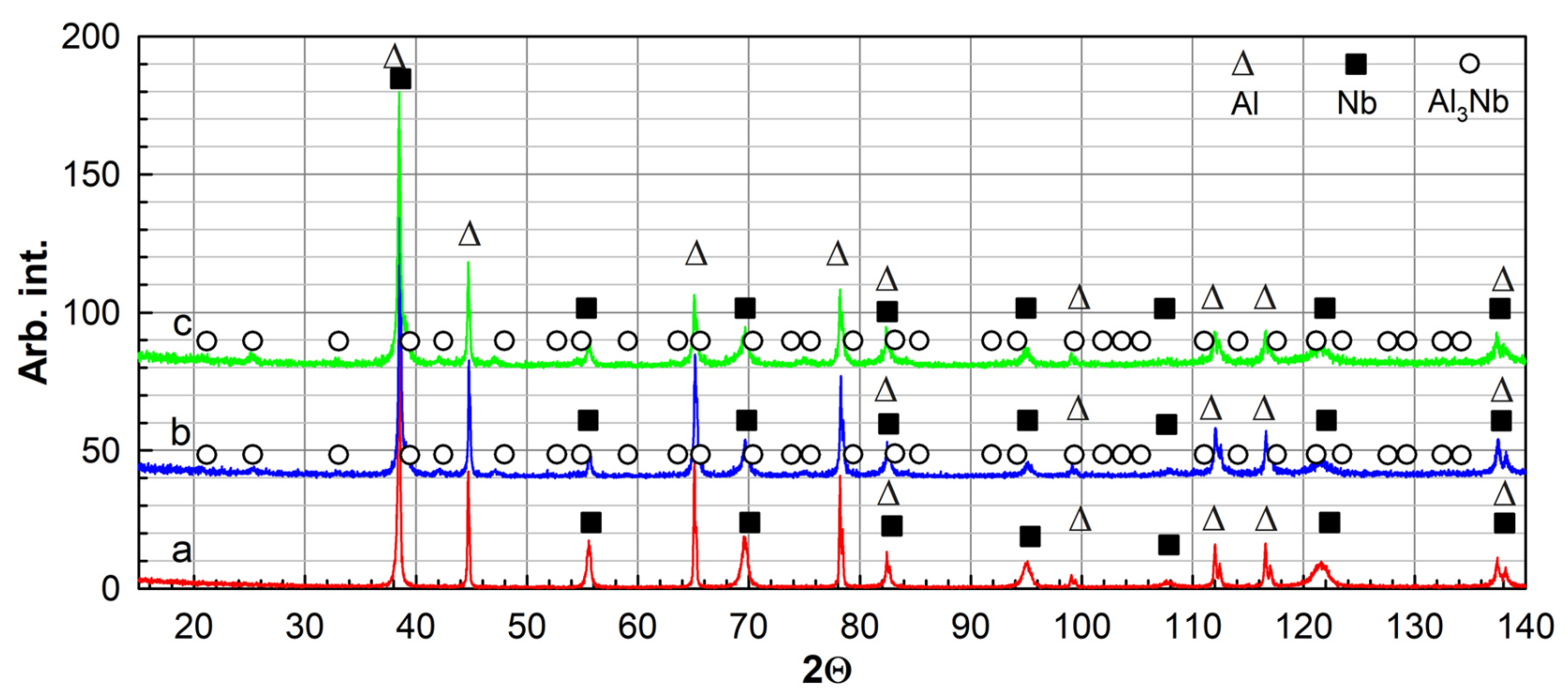

Pис. 3. (Color online) Рентгенограммы с образцов композиционного материала $\mathrm{Al}-\mathrm{Nb}-\mathrm{Al} \mathrm{c}$ диаметром $\mathrm{Al}$ диска 10 мм, изготовленных ИПДК при $\omega=2$ об/мин и $N=10$ (a); $N=25$ (b); $N=30$ (c).

Fig. 3. (Color online) X-Ray diffraction pattern of $\mathrm{Al}-\mathrm{Nb}-\mathrm{Al}$ samples with diameter $\mathrm{Al}$ disk $10 \mathrm{~mm}$, processed by HPT at $\omega=2 \mathrm{rpm}$ and $N=10$ (a); $N=25$ (b); $N=30$ (c).

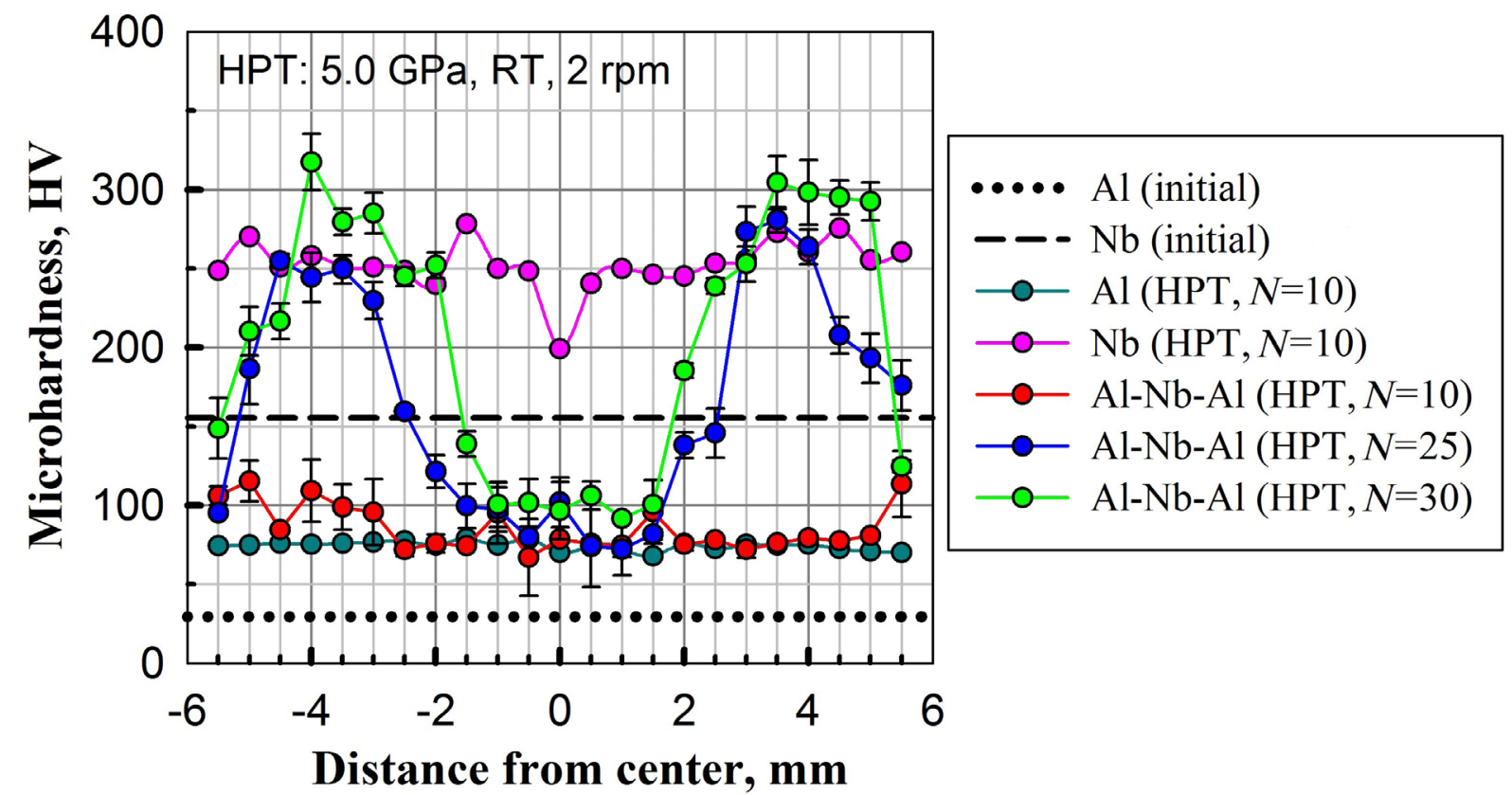

Pис. 4. (Color online) Изменение микротвердости вдоль поперечного диаметрального сечения исходных и деформированных образцов алюминия, ниобия и трехслойных пакетов Al-Nb-Al (с диаметром диска алюминия 10 мм).

Fig. 4. (Color online) The microhardness change along the diameter cross section of the initial and deformed samples of aluminum, niobium, and $\mathrm{Al}-\mathrm{Nb}-\mathrm{Al}$ three-layer packets (with initial diameters of an aluminum disk $10 \mathrm{~mm}$ ).

\section{4. Заключение}

Методом интенсивной пластической деформации кручением под высоким давлением трехслойного пакета из чередующихся дисков $\mathrm{Al}-\mathrm{Nb}-\mathrm{Al}$ были сформированы монолитные и бездефектные образцы алюмоматричного композита гибридной системы при всех исследованных режимах деформации. При этом экспериментально подобрано оптимальное сочетание диаметров диска из $\mathrm{Al}$, с точки зрения повышения технологической пластичности (10 мм), и $\mathrm{Nb}$ образца (12 мм). Максимальное дробление и распределение $\mathrm{Nb}$ в $\mathrm{Al}$ наблюдаются при скорости деформации 2 об/мин. Расширение области наибольшего измельчения структурных составляющих и формирования металломатричной структуры имеют место при количестве оборотов 25 и 30. Кроме того, ИПД способствует формированию в образце композиционного материала тонкодисперной пластинчатой 
микроструктуры на середине радиуса и однородного распределения $\mathrm{Nb}$ в $\mathrm{Al}$ матрице на периферии, в центpe сохраняются отдельные изогнутые слои $\mathrm{Nb}$ в $\mathrm{Al}$. При этом методом рентгеноструктурного анализа продемонстрирован синтез упрочняющей интерметаллидной фазы $\mathrm{Al}_{3} \mathrm{Nb}$ за счет протекания процессов деформационно-индуцированного старения. Изменение микротвердости по диаметру образца алюмоматричного композита согласоются с изменениями микроструктуры. В центре образцов микротвердость минимальна и находится на уровне около $100 \mathrm{HV}$, на середине достигает максимальных значений около 280 и $300 \mathrm{HV}$ и на периферии составляет около $130 \mathrm{HV}$, соответственно для $N=25$ и $N=30$.

Благодарности/Acknowledgements. Работа выполнена в рамках государственного задания ИПСМ РАН и поддержана РНФ (грант № 18-12-00440). Экспериментальные исследования выполнены на базе ЦКП ИПСМ РАН. / The present work was accomplished according to the state assignment of IMSP RAS and supported by the Russian Science Foundation (Grant No. 18-12-00440). Experimental studies were carried out on the facilities of shared services center of IMSP RAS.

\section{Литература/References}

1. R.S. Mishra, Z.Y. Ma. Mater. Sci. Eng. R. 50, 1 (2005). Crossref

2. C. Suryanarayana. Madridge J. Nanotechnol. Nanosci. 4 (1), 127 (2019). Crossref

3. T. S. Srivatsan, T. Sudarshan, E. Lavernia. Prog. Mater. Sci. 39, 317 (1995). Crossref

4. S. R. H. Zeidabadi, H. Daneshmanesh. Mater. Sci. Eng. A. 702, 189 (2017). $\underline{\text { Crossref }}$

5. G. R. Khalikova, G. F. Korznikova, V.G. Trifonov. Lett. Mater. 7 (1), 3 (2017). (in Russian) [Г.Р. Халикова, Г.Ф. Корзникова, В.Г. Трифонов. Письма о материалах. 7 (1), 3 (2017).] Crossref

6. V. Viswanathan, T. Laha, K. Balani, A. Agarwal, S. Seal. Mater. Sci. Eng. R. 54 (5-6), 121 (2006). Crossref
7. J.-K. Han, K.-D. Liss, T. G. Langdon, M. Kawasaki. Sci. Rep. 9, 17186 (2019). $\underline{\text { Crossref }}$

8. G.F. Korznikova, K.S. Nazarov, R. Kh. Khisamov, S.N. Sergeev, R.U. Shayachmetov, G.R. Khalikova, J. A. Baimova, A. M. Glezer, R. R. Mulyukov. Mater. Lett. 253, 412 (2019). Crossref

9. M. Pouryazdan, B. J. P. Kaus, A. Rack, A. Ershov, H. Hahn. Nat. Commun. 8, 1611 (2017). Crossref

10. D. Hernández-Escobar, Z.U. Rahman, H. Yilmazer, M. Kawasaki, C. J. Boehlert. Phil. Mag. 99 (5), 557 (2019). $\underline{\text { Crossref }}$

11. M. Robert, S. Urtiga Filho. J. Mater. Process. Technol. 64 (1-3), 335 (1997). $\underline{\text { Crossref }}$

12. J. Mazumder, H. Chung, T. Yamamoto, T. Duffey, H. Sehitoglu, M. Jilavi, W. M. Kriven. Nanostruct. Mater. 9, 75 (1997). Crossref

13. G.F. Korznikova, R. R. Mulyukov, A.M. Zhilyaev, V.N. Danilenko, R.Kh. Khisamov, K.S. Nazarov, S. N. Sergeyev, G. R. Khalikova, R. R. Kabirov. AIP Conf. Proc. 2053, 030028 (2018). $\underline{\text { Crossref }}$

14. R.Z. Valiev, A.P. Zhilyaev, T.G. Langdon. Bulk nanostructured materials: Fundamentals and applications. Wiley-Blackwell (2013) 440 p. Crossref

15. J. G. Li, M. Umemoto, Y. Todaka, K. Fujisaku, K. Tsuchiya. Rev. Adv. Mater. Sci. 18, 577 (2008).

16. Y. Cao, M. Kawasaki, Y. B. Wang, S. N. Alhajeri, X. Z. Liao, W. L. Zheng, S. P. Ringer, Y. T. Zhu, T. G. Langdon. J. Mater. Sci. 45, 4545 (2010). Crossref

17. Y. Cao, Y. B. Wang, R. B. Figueiredo, L. Chang, X. Z. Liao, M. Kawasaki, W.L. Zheng, S.P. Ringer, T. G. Langdon, Y.T. Zhu. Acta Mater. 59, 3903 (2011). Crossref

18. G. F. Korznikova, T.H. Czeppe, A. V. Korznikov. Lett. Mater. 4 (2), 117 (2014). Crossref

19. R. Kulagin, Y. Beygelzimer, Yu. Ivanisenko, A. Mazilkin, B. Straumal, H. Hahn. Mater. Lett. 222, 172 (2018). Crossref

20. ASM Handbook. Vol. 3: Alloy Phase Diagrams (Ed. by H. Okamoto, M.E. Schlesinger, E.M. Mueller). ASM International (2016) 800 p.

21. R. Pretorius, A. Vredenberg, F. Saris, R. De Reus. J. Appl. Phys. 70, 3636 (1991). 당s 